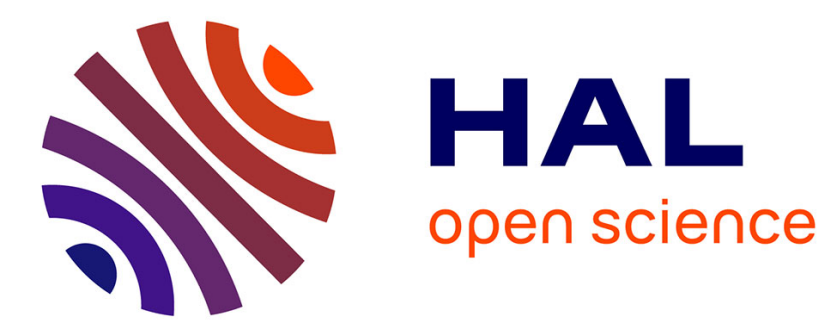

\title{
A Modified 2D Visco-Acoustic Waveform Inversion Scheme
}

\author{
Hao Jiang, Hervé Chauris
}

\section{To cite this version:}

Hao Jiang, Hervé Chauris. A Modified 2D Visco-Acoustic Waveform Inversion Scheme. 80th EAGE

Conference and Exhibition 2018, Jun 2018, Copenhagen, Denmark. hal-01884679

\section{HAL Id: hal-01884679 \\ https://hal.science/hal-01884679}

Submitted on 1 Oct 2018

HAL is a multi-disciplinary open access archive for the deposit and dissemination of scientific research documents, whether they are published or not. The documents may come from teaching and research institutions in France or abroad, or from public or private research centers.
L'archive ouverte pluridisciplinaire HAL, est destinée au dépôt et à la diffusion de documents scientifiques de niveau recherche, publiés ou non, émanant des établissements d'enseignement et de recherche français ou étrangers, des laboratoires publics ou privés. 


\section{Th A12 07}

\section{A Modified 2D Visco-Acoustic Waveform Inversion Scheme}

H. Jiang* (MINES ParisTech, PSL Research University), H. Chauris (MINES ParisTech, PSL Research University)

\section{Summary}

Seismic attenuation and velocity are two coupled parameters with different sensitivities. We propose to explicitly use the dispersion relation during full waveform inversion. In a first step, a classical visco-acoustic inversion is applied for a fixed quality factor, then a subsequent modified full waveform inver- sion leads to a faster convergence compared to the standard scheme. The approach is validated on a simple 2D synthetic data set mainly dominated by transmitted waves. The new scheme is an alternative to the Hessian-based strategy. 


\section{Introduction}

Seismic attenuation is a very useful property to detect fluid beyond the traditional resolution of velocityrelated seismic images in exploration geophysics (Brzostowski and McMechan, 1992). The estimation of the $Q$ factor can be split into two main methods: traveltime tomography and wave-equation based methods. The first kind of methods analyses the amplitude loss or the spectrum distortion (Brzostowski and McMechan, 1992; Leggett et al., 1992; Zucca et al., 1994; Quan and Harris, 1997; Plessix, 2006). For the alternative approaches, it relies on the Full Waveform Inversion (FWI), minimising the misfit between computed and observed data (Barnes et al., 2014; Li et al., 2015; Gao and Wang, 2016).

Velocity and attenuation are coupled and both parameters can not be retrieved independently from surface data. Different strategies have been developed (Kamei and Pratt, 2013; Gao and Wang, 2016; Plessix et al., 2016). The first possibility consists of first determining the velocity parameters, for a fixed $Q$ factor, and in a second phase of inverting the two parameters, as the velocity model is better constrained. This sequential approach may lead to data over-fitting. In the second strategy, velocity and attenuation are estimated simultaneously with the introduction of the Hessian (second-order derivative of the objective function), either in a quasi-Newton approach (Virieux, 2017) or with a truncated Newton approach (Métivier, 2014). The role of the Hessian is to deal with the different sensitivities and coupling between velocity and attenuation.

The dispersion of viscous waves leads to phase velocity modifications. Because of the band-limited aspect of seismic waves, different combinations of velocity and $Q$ parameters can have the same equivalent kinematic velocity information. In this work, we propose a modified full waveform inversion algorithm, based on the sequential approach, to explicitly take into account the dispersion relation. The objective is to handle the coupling between velocity and attenuation, without relying on the Hessian. We first indicate how we model attenuation, then present the modified FWI approach. It mainly consists of modifying the gradient of the objective function. We compare the behaviours of the classical and new schemes on $2 \mathrm{~d}$ inversions on a synthetic data set, and conclude with the recommended strategy for inverting velocity and attenuation.

\section{Theory}

For the time-domain visco-acoustic wave-equation, the nearly constant $Q$ theory by Futterman (1962), involves a time convolution in the stress-strain relation, indicated by the Standard Linear Solid equation (SLS)

$$
Q(\omega)=\frac{\operatorname{Re}[M(\omega)]}{\operatorname{Im}[M(\omega)]}=\frac{1-L+\sum_{l=1}^{L} \frac{1+\omega^{2} \tau_{\varepsilon l} \tau_{\sigma l}}{1+\omega^{2} \tau_{\sigma l}^{2}}}{\sum_{l=1}^{L} \frac{\omega\left(\tau_{\varepsilon l}-\tau_{\sigma l}\right)}{1+\omega^{2} \tau_{\sigma l}^{2}}},
$$

where $M(\omega)$ is the complex modulus, and $\tau_{\sigma l}$ and $\tau_{\varepsilon l}$ are the stress and strain relaxation times for the $l$ th SLS. L is number of SLS. To optimise constant $Q$ within a frequency range, $\tau_{\sigma l}$ is given in advance. Usually, distributing the $\tau_{\sigma l}$ logarithmically over the frequency range of interest yields good approximations to a constant $Q$ with $\tau_{\sigma l}=1 / \omega_{l}$, about one per one-two octaves. Then, $L$ parameters $\tau_{\varepsilon l}$ are optimised to have constant $Q$. To simplify the implementation, Blanch (1995) uses only one parameter $\tau=\tau_{\varepsilon l} / \tau_{\sigma l}-1$ to replace parameters $\tau_{\varepsilon l}$. With the single $\tau$ parameter, the visco-acoustic wave-equation reads

$$
\begin{aligned}
\frac{\partial p}{\partial t}+\kappa(L \tau+1)(\nabla \cdot \vec{v})+\sum_{l=1}^{L} r_{p}^{l} & =f\left(x_{s}, t\right), \\
\frac{\partial \vec{v}}{\partial t}+\frac{1}{\rho} \nabla p & =0, \\
\frac{\partial r_{p}^{l}}{\partial t}+\frac{1}{\tau_{\sigma}^{l}}\left(r_{p}^{l}+\tau \kappa(\nabla \cdot \vec{v})\right) & =0, \quad l=\in[1, L]
\end{aligned}
$$

where $l$ means the $l$ th SLS among $L$ to represent the constant $Q$ factor. $p$ is acoustic pressure field, $\kappa=\rho v_{p}^{2}$ is the relaxed modulus. To analyse the visco-acoustic phase velocity in details, we rewrite the 
wave equation in frequency domain as a second-order differential equation

$$
(i \omega)^{2} p-v_{\text {phase }}^{2} \Delta p=i \omega f\left(x_{s}, \omega\right),
$$

with a complex phase velocity

$$
v_{\text {phase }}^{2}=v_{p}^{2}\left[(L \tau+1)-\sum_{l=1}^{L} \frac{\tau}{1+i \omega \tau_{\sigma}^{l}}\right] .
$$

The phase velocity can be split into a real term and an imaginary part

$$
v_{\text {phase }}=v_{k}\left(v_{p}, \tau, \omega\right)+i v_{l}\left(v_{p}, \tau, \omega\right),
$$

where $v_{k}$ and $v_{l}$ are the real and imaginary parts of the phase velocity, associated to the kinematic part and the energy loss, respectively. We call $v_{k}$ as the equivalent kinematic velocity, which is the function of $v_{p}, \tau$ and frequency $\omega$. For the same frequency, different combinations of $v_{p}$ and $\tau$ can have same $v_{k}$. For example, $v_{k}$ is $2250 \mathrm{~m} / \mathrm{s}$ at $20 \mathrm{~Hz}$. Combinations of $v_{p}$ and $\tau$ for a given $v_{k}$ are linked according to the curve in Figure 1. It indicates that, waves with different $v_{p}$ and $\tau$ have the same traveling time but amplitude differences.

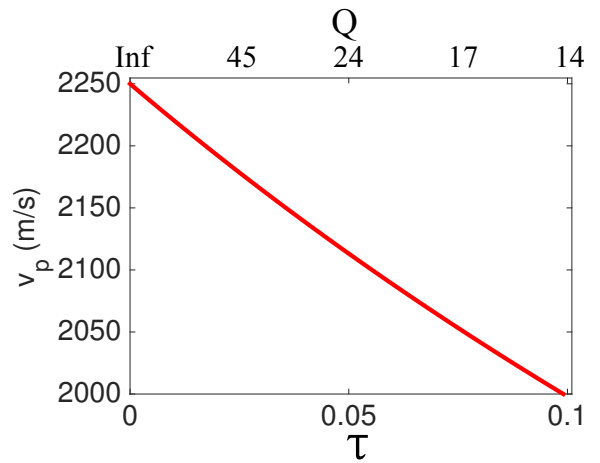

Figure 1 Different combinations of $v_{p}$ and $\tau$, leading to the same $v_{k}=2250 \mathrm{~m} / \mathrm{s}$ at reference frequency $20 \mathrm{~Hz}$.

We propose to modify the classical FWI objective function $J\left(v_{p}\right)$ by introducing the dependency of $v_{p}$ over $\tau$. The total derivative reads

$$
\frac{d J\left(v_{p}\right)}{d v_{p}}=\frac{\partial J\left(v_{p}\right)}{\partial v_{p}}+\frac{\partial \tau\left(v_{p}\right)}{\partial v_{p}} \frac{\partial J\left(v_{p}\right)}{\partial \tau} .
$$

The derivatives of $J$ with respect to $v_{p}$ and $\tau$ can be obtained with the adjoint state technique.

\section{Inversion strategy and results}

We compare three different strategies all based on a non-linear conjugate gradient approach:

- Strategy A is a pure acoustic inversion, where only $v_{p}$ is updated, while $\tau$ (or $Q$ ) remains fixed, here set to infinity;

- Strategy B is the classical visco-acoustic FWI where $v_{p}$ and $\tau$ (or $Q$ ) are simultaneously inverted with a non-linear conjugate gradient, without any Hessian information;

- Strategy $\mathrm{C}$ is the modified viso-acoustic FWI, where $\tau$ (or $Q$ ) is deduced from $v_{p}$ everytime the velocity is updated, according to Equation 7, with a modified gradient (Equation 8).

We consider sources at the surface and receivers in a horizontal well at $200 \mathrm{~m}$ depth. 61 sources are located between 200 and $440 \mathrm{~m}$, every $4 \mathrm{~m}$, while receivers are deployed between 0 and $640 \mathrm{~m}$. The 
exact model is homogeneous with $v_{p}=2000 \mathrm{~m} / \mathrm{s}$ and $Q=50$ (or $\tau=0.023$ ) in Figures 3a and 3b. The equivalent kinematic velocity for a central frequency at $20 \mathrm{~Hz}$ is $2060 \mathrm{~m} / \mathrm{s}$.

The initial model is also homogeneous, with $v_{p}=2150 \mathrm{~m} / \mathrm{s}$ and $Q$ is set to infinity (or $\tau=0$ ) in Figures $3 \mathrm{c}$ and $3 \mathrm{~d}$. In a first phase, we perform a pure acoustic FWI and only update $v_{p}$ (strategy A). The objective function decreases from 1.00 to 0.08 (Figure 2, red curve) and cannot go below as the quality factor is not updated. The final $v_{p}$ model is around $2060 \mathrm{~m} / \mathrm{s}$ and fails in a local minimum (Figure $3 \mathrm{~g}$ ). For the 3rd iteration, the velocity is already around the kinematic velocity $2060 \mathrm{~m} / \mathrm{s}$ (Figure 3e). With the visco-acoustic inversion, more than 20 iterations are needed with the standard B strategy (Figure 3, green curve). After 30 iteration, the velocity is around the correct $v_{p}=2000 \mathrm{~m} / \mathrm{s}$ and $\tau=0.023$ (or $Q=50)$ in Figures $3 \mathrm{i}$ and $3 \mathrm{j}$.

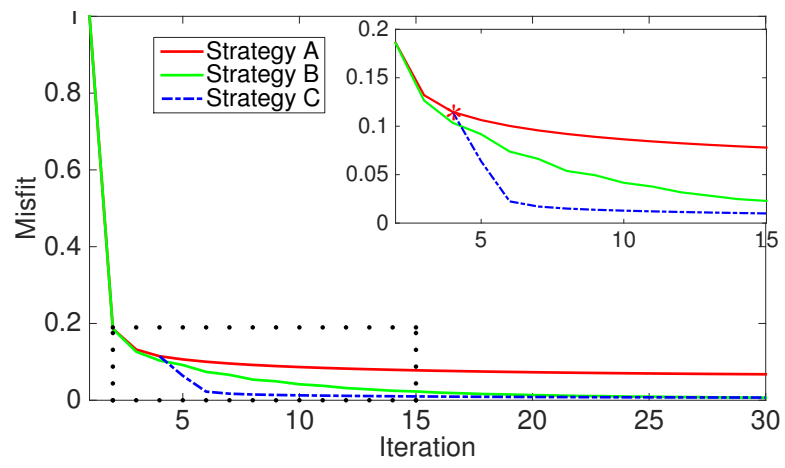

Figure 2 Misfit comparison for three different strategies, strategy A (red), strategy B (green), and strategy C (blue).

Starting from the same models, we first apply three iterations with the A-strategy, followed by 3 other iterations with the new C-strategy. The convergence rate for the new approach (Figure 2, blue line) is faster than the classical FWI approach (Figure 2, green line). The final results show that the inverted velocity is around the correct $v_{p}=2000 \mathrm{~m} / \mathrm{s}$ and $\tau=0.023$ (or $Q=50$ ) in Figures $3 \mathrm{k}$ and 31 . It is comparable to the conventional result after 30 iterations. Comparing strategy B with C, our new FWI converges faster than conventional visco-acoustic FWI for the same final quality. The velocity derivative with respect to $\tau$ acts as a balancing parameter to adjust the sensitivity of velocity and $\tau$ gradients. An alternative approach would be to use the information for the Hessian (Virieux, 2017).

\section{Conclusions}

For conventional visco-acoustic inversion, where velocity and attenuation are simultaneously inverted, the two parameters are coupled, with different sensitivities. A first approach consists of introducing the Hessian. Here, we propose to directly incorporate the phase velocity dispersion relation in the minimisation process: an update on $v_{p}$ also leads to a modification of the attenuation factor. The strategy is applied after a few iterations with a pure acoustic inversion. The convergence appears to be faster that the standard visco-acoustic full waveform approach. In the future, the new approach should be compared to FWI with the incorporation of the Hessian.

\section{Acknowledgements}

The research described in this paper was carried out as a part of the Paris Exploration Geophysics Group project (GPX) funded by the French National Research Agency, CGG, TOTAL and Schlumberger.

\section{References}

Barnes, C., Charara, M. and Williamson, P. [2014] P and S Wave Attenuation Effects on Full-Waveform Inversion for Marine Seismic Data. In: Expanded Abstracts. 84th SEG Technical Program, 949-953.

Blanch, J.O., Robertsson, J.O.A. and Symes, W.W. [1995] Modelling of a constant Q: Methodology and algorithm for an efficient and optimally inexpensive viscoelastic technique. Geophysics, 60, 176-184. 


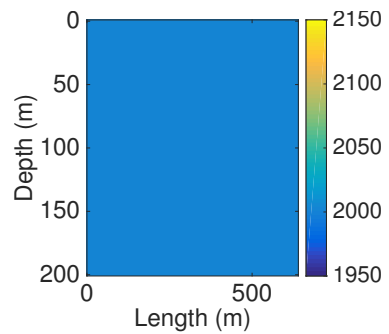

(a)

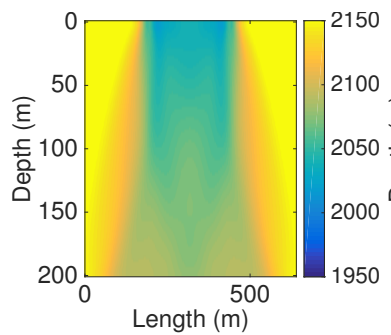

(e)

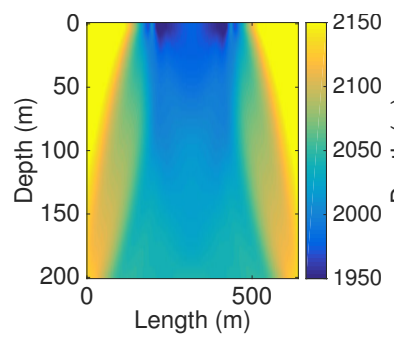

(i)

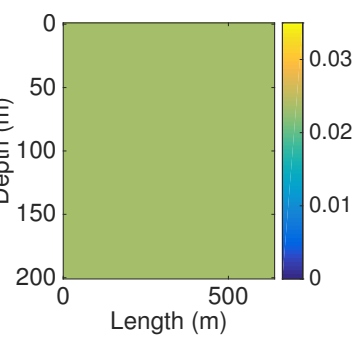

(b)

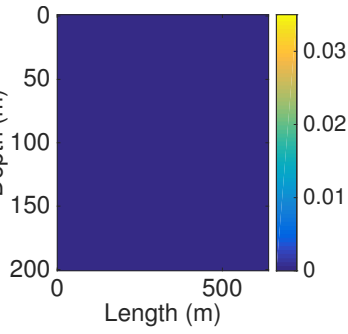

(f)

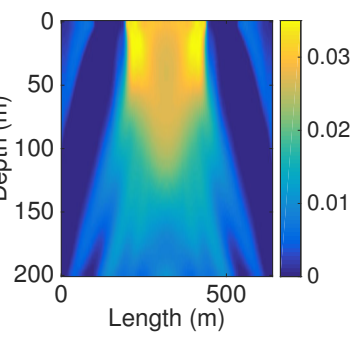

(j)

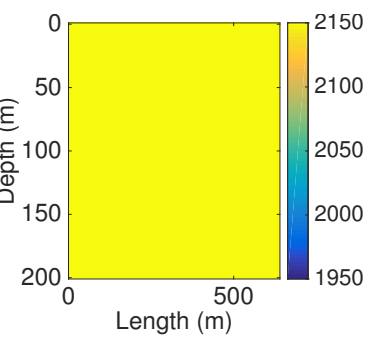

(c)

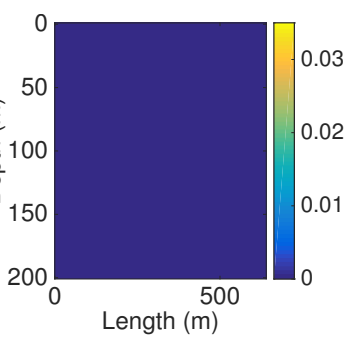

(d)

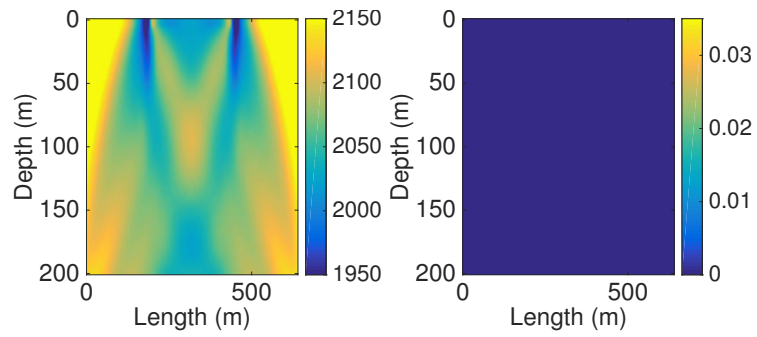

(g)

(h)

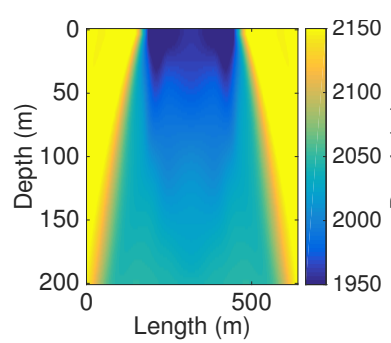

(k)

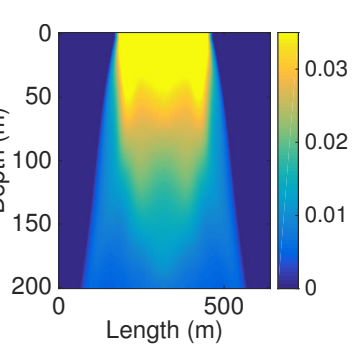

(l)

Figure 3 a and b: exact velocity and $\tau$ models, $\mathrm{c}$ and d: initial velocity and $\tau$ models, e and f: updated velocity models after 3 iterations (strategy $\mathrm{A}$ ), $\mathrm{g}$ and h: updated velocity model after 30 iterations (strategy A), $\mathrm{i}$ and $\mathrm{j}$ : updated velocity and $\tau$ models after 30 iterations (strategy B), $\mathrm{k}$ and $\mathrm{l}$ : updated velocity and $\tau$ models after 3 iterations (strategy $\mathrm{C}$ ).

Brzostowski , M. and McMechan, G. [1992] 3-D tomographic imaging of near-surface seismic velocity and attenuation. Geophysics, 57, 396-403.

Futterman, W.I. [1962] Dispersive body wave. J. Geophys. Res., 67, 5279-5291.

Gao, F. and Wang, Y. [2016] Simultaneous inversion for velocity and attenuation by waveform tomography. Journal of applied geophysics, 131, 103-108.

Kamei, R. and Pratt, R.G. [2013] Inversion strategies for visco-acoustic waveform inversion. Geophys. J. Int., 859-884.

Leggett, M., Goulty, N.R. and Kragh, J.E. [1992] Study of traveltime and amplitude time-lapse tomography using physical model data. In: Expanded Abstracts. 54th Annual EAGE Meeting and Exposition, 248-249.

Métivier, L., Bretaudeau, L., Brossier, R., Operto, S. and , J. [2014] Full waveform inversion and truncated Newton method: quantitative imaging of complex subsurface sturctures. Geophysical Prospecting, 62(6), 1203-1483.

Plessix, R.-E. [2006] Estimation of velocity and attenuation coefficient maps from crosswell seismic data. Geophysics, 71(6), S235-S240.

Plessix, R.E., Stopin, A., Kuehl, H., Goh, V. and Overgaag, K. [2016] Visco-acoustic full waveform inverision. In: Expanded Abstracts. 54th Annual EAGE Meeting and Exposition.

Quan, Y. and Harris, J. [1997] Seismic attenuation tomography using the frequency shift method. Geophysics, 62(3), 895-905.

Virieux, J. [2017] Multiparameter FWI using matrix-free truncated Newton approach for time-domain visco-acoustic case. In: SEG workshop. 3rd Full Waveform inversion: What are we getting?

Zucca, J.J., Hutchings, L.J. and Kasameyer, L.J. [1994] Seismic velocity and attenuation structure of the Geysers geothermal field, California. Geothermics, 23, 111-126. 